Title:

\title{
Invasive Candidiasis in Critical Care: Challenges and Future Directions
}

Logan $\mathrm{C}^{1,2}$, Martin-Loeches $\mathrm{I}^{3,4}$, Bicanic $\mathrm{T}^{1,2}$.

${ }^{1}$ Institute of Infection and Immunity, St George's University of London, United Kingdom

${ }^{2}$ Clinical Infection Unit, St George's University Hospital, London, United Kingdom

${ }^{3}$ Multidisciplinary Intensive Care Research Organization (MICRO), St. James's Hospital, Dublin, Ireland

${ }^{4}$ Hospital Clinic, Universidad de Barcelona, CIBERes, Barcelona, Spain

\section{Running title:}

Invasive Candidiasis in Critical Care

\section{Corresponding autor:}

Ignacio Martin-Loeches

Email: drmartinloeches@gmail.com

Narrative review

Word count (excluding tables, figures and legends): 4,000 


\section{ABSTRACT}

Invasive candidiasis is the most common critical care-associated fungal infection with a crude mortality of $\sim 40-55 \%$. Important factors contributing to risk of invasive candidiasis in ICU include use of broad-spectrum antimicrobials, immunosuppressive drugs, and total parenteral nutrition alongside iatrogenic interventions which breach natural barriers to infection (vascular catheters, renal replacement therapy, Extra Corporeal Membrane Oxygenation (ECMO), surgery). This review discusses three key challenges in this field. The first is the shift in Candida epidemiology across the globe to more resistant non-albicans species, in particular, the emergence of multiresistant Candida glabrata and Candida auris, which pose significant treatment and infection control challenges in critical care. The second challenge lies in timely and appropriate initiation and discontinuation of antifungal therapy. Early antifungal strategies (prophylaxis, empirical and pre-emptive) using tools such as the Candida colonisation index, clinical prediction rules and fungal non-culture-based tests have been developed: we review the evidence on implementation of these tools in critical care to aid clinical decision-making around the prescribing and cessation of antifungal therapy. The third challenge is selection of the most appropriate antifungal to use in critical care patients. While guidelines exist to aid choice, this heterogenous and complex patient group require a more tailored approach, particularly in cases of acute kidney injury, liver impairment and for patients supported by Extra Corporeal Membrane Oxygenation. We highlight key research priorities to overcome these challenges in the future.

\section{$\underline{\text { Kevwords }}$}

critical care; candidiasis; fungal; biomarkers; antifungal

\section{Declarations}

\section{Funding None}

\section{Conflicts of interest}

IML is a section-editor for Intensive Care Medicine and has received speaking and advisory board fees from Merck and Gilead Sciences. TB has received speaking fees from Pfizer and speaking, advisory board fees and research support from Gilead Sciences. CL has received research support from Gilead Sciences. 


\section{Authors' Contributions}

CL, TB and IML conceived the idea, CL performed the literature search and drafted the manuscript, which was critically reviewed and revised by IML and TB.

Take home message: Epidemiological shifts towards multi-resistant Candida requires enhanced surveillance and rigorous infection control to detect and prevent resistance emergence. The evidence around deployment of risk-scores and fungal non-culture-based tests in decisionmaking around starting and stopping antifungals in the ICU is lacking: adequately powered multisite studies using a combination of tests linked to clinical and cost effectiveness outcomes are needed. Antifungal prescribing in special ICU populations, particularly acute kidney injury, liver impairment and ECMO requires a tailored approach and further PK evaluation.

Tweet: Key ICU Candidiasis Challenges: Resistance Emergence, Biomarker-driven antifungal prescribing (start \& stop), Tailoring therapy in ICU hosts 


\section{Introduction}

'Invasive candidiasis' (IC) is an umbrella term for three clinical conditions; candidemia; deepseated candidiasis; and deep-seated candidiasis with associated candidemia[1]. Cases are often hospital-acquired, and critically ill patients are particularly vulnerable[2], with approximately onethird of all candidemia occurring in this setting[3]. Despite expanded access to fungicidal agents, IC-related outcomes remain poor, with a crude mortality of $\sim 40-55 \%$ in Intensive Care Unit (ICU)focused studies over the past decade[4-7].

The incidence of deep-seated candidiasis without concomitant candidaemia in ICU is less certain due to challenges in obtaining specimens for microbiological confirmation. Intra-abdominal candidiasis (IAC) accounts for most deep-seated cases, with $\sim 30 \%$ occurring in critical care[8]. Perforation, anastomotic leaks, repeat laparotomies, necrotizing pancreatitis and abdominal organ transplants increase risk, therefore incidence is higher in surgical ICUs[8]. Other forms of deep-seated candidiasis include haematogenously disseminated disease (hepatosplenic, ocular, cardiac, central nervous system, bone and renal), seen more frequently with prolonged candidemia, and in immunosuppressed and neutropenic patients[9, 10]. Host genetics also influence IC susceptibility, with various single-nucleotide polymorphisms (SNPs) identified as increasing candidaemia risk[11].

Figure 1. illustrates key factors contributing to development of IC in ICU. IC risk factors have fluctuated with advances in intensive care medicine; while there is increased use of renal replacement therapy, Extra Corporeal Membrane Oxygenation (ECMO), and immunosuppression treatments, there has been improved vascular catheter management, more judicious use of total parenteral nutrition (TPN) and greater emphasis on antimicrobial stewardship[12-14]. The collective impact of this on IC incidence is unclear. Large multi-centre studies examining IC incidence in ICU have been conducted over the past decade[4-7, 15-18]. Rates of candidaemia reported vary significantly between 3.5 - 16.5 per 1000 admissions[4, 6, 7, 16-18]. However, due to inter-centre variability, the fact most studies focused on candidaemia only, and some encompassed cases likely to represent colonization rather than IC, evaluation of IC incidence trends in ICU over time is challenging.

In this narrative review, we sought to summarise key epidemiological, diagnostic and treatment challenges of managing IC in ICU and highlight future directions in this field. To ensure broad coverage of relevant literature, we undertook a MEDLINE search for English language articles published before 1 July 2020, using the terms "candidiasis", "candidaemia", "critical care", 
"resistance", "biomarkers" and "antifungal", including further relevant studies from reference lists of articles identified. 


\section{Challenge 1: Changing Epidemiology and Emergence of Antifungal Resistance}

Epidemiological shifts

There is significant geographic and demographic variation in IC[19]. C. albicans remains the dominant species in Europe[5-7]; in a pan-European ICU cohort study (2015-16)[6], C. albicans represented $57 \%$ of cases, followed by C. glabrata and C. parapsilosis. Across India, C. tropicalis was the most common cause of ICU-acquired candidaemia[20], whereas $C$. albicans and $C$. parapsilosis predominate in Latin America[21]. The USA sees a higher proportion of non-albicans cases (approximately two-thirds), with increasing C. glabrata incidence[22]. Echinocandinresistant $C$. glabrata is reported; while European prevalence appears low (<1\%)[23], US studies report a prevalence of 6-12\%[24-26], with azole cross-resistance in up to one-third of isolates[27]. This is concerning given echinocandins are recommended first-line treatment in IC, and azoles the most widely used antifungals globally. Moreover, the emergent multi-drug resistant $C$. auris has caused outbreaks on ICUs worldwide[28]. It is the $3^{\text {rd }}$ most common cause of candidaemia in South Africa, with $88 \%$ of cases associated with ICU stays[29]. C.auris is usually fluconazoleresistant, with variable amphotericin and echinocandin susceptibility, and pan-fungal resistance to all three classes reported[30].

\section{Reservoirs of resistance in ICU: the patient and environment}

The patient and the environment can be reservoirs of fungal resistance in ICU. Antibiotic use disrupts the skin and gut microbiome, increasing Candida colonization and risk of IC[31]. Antifungal exposure selects for less susceptible Candida species such as $C$. parapsilosis, $C$. krusei and C. glabrata[32] and fosters resistance; in a US study, echinocandin-resistant $C$. glabrata was associated with prior echinocandin exposure, fluconazole resistance, and prolonged hospitalization[26]. In Denmark, post-treatment ( $\geq 7$ days) mouth swabs in candidaemic patients demonstrated acquired resistance to fluconazole and echinocandins in $29 \%$ and $22 \%$ of C.glabrata isolates respectively[33]. Specifically, reduced echinocandin penetration into the gut may select for the emergence of echinocandin-resistant species[33, 34].

Resistant isolates spread between patients, and within the ICU environment, with reports of genotype-linked clusters of azole-resistant C. parapsilosis[35], and inter-hospital spread of azoleresistant $C$. glabrata[36] in ICU. C. auris studies have described widespread contamination of environmental surfaces and equipment persisting for months, with patient acquisition of $C$. auris occurring after as little as 4-hours of contact[37]. The limited efficacy of commonly used environmental disinfectants and absence of effective skin decolonization regimens for $C$. auris, 
have made transmission difficult to interrupt[38]. A UK ICU C.auris outbreak was only stemmed when reusable temperature probes were removed from circulation[39]. For $C$. auris, infection control measures including screening, isolation, cohorting and environmental disinfection are advised in Public Health guidance[40, 41]. ICU interventions for tackling fungal resistance are summarized in Figure 2.

Many hospital laboratories do not identify yeasts in non-sterile specimens to species level; as a result, changes in ecology and resistance may go undetected. Misidentification of $C$. auris for other species, particularly C. haemulonii, when using common diagnostic platforms is recognized[42]. Matrix-assisted laser desorption/ionization time-of-flight (MALDI-TOF) allows quick, accurate identification but is not universally available. Improving laboratory capacity for Candida speciation, particularly C.auris, and fungal susceptibility testing is important for surveillance and early detection of resistance emergence in ICU. 


\section{Challenge 2: When to Start and When to Stop Antifungal Therapy?}

Timely delivery of effective AFT in proven IC is crucial, as delays are associated with increased mortality[43]. Conversely, over-prescription may be detrimental, exposing patients to drug toxicities and driving resistance emergence. A cross-sectional study of French and Belgian ICUs demonstrated that while $7.5 \%$ of patients were prescribed systemic AFT, two-thirds subsequently had no evidence of IFI[44], emphasising the challenge of achieving a balance between targeted, timely AFT whilst avoiding excessive and unnecessary use.

Earlier antifungal strategies have thus been developed (prophylactic, empiric, pre-emptive), (Table 1) although the optimal strategy in ICU remains controversial. To aid decision-making about stopping and starting antifungals, three key tools, for use alone or in combination, have been proposed: Candida colonisation assessment, clinical prediction rules, and fungal nonculture-based tests (NCBT) (Table 2). Table 3 summaries key studies using these tools to initiate or discontinue AFT, however their impact on clinical practice remains hotly debated.

\section{Role of candida colonization and clinical prediction rules}

Candida colonization is considered a pre-requisite for the development of IC (Fig.1)[62]; those with a higher Candida Colonization Index $(\mathrm{CCl})$ are at greater risk[45]. However, although the proportion of ICU patients colonized with Candida increases over time ( $50-80 \%)$, only 5-30\% will develop IC[63]. While studies have proposed colonization can be used to guide prophylaxis and reduce IC[64-66], they have not shown a mortality benefit. A study found colonization-triggered caspofungin or azole use changed the ICU fungal ecology (increased C. glabrata), without reducing IC-associated mortality or incidence[67]. Hence, the moderate positive predictive value (PPV) of this approach ( 66\% for CCI[45]) could lead to excessive antifungal use that is neither appropriate or cost-effective.

To improve the PPV, clinical prediction rules, incorporating host factors with or without Candida colonization, have been established. The UK FIRE Study reviewed 60,000 ICU admissions and evaluated risk models for predicting IC. However, IC incidence was lower than expected (0.6\%), and analysis suggested a strategy of no risk-assessment or AFT prophylaxis was the most costeffective [68].

RCTs have evaluated the impact of clinical prediction rules triggering early AFT on IC incidence and mortality in ICU. The MSG-01 trial[57] $(n=219)$ randomized to caspofungin prophylaxis or placebo based on the Ostrosky-Zeichner Clinical Prediction Rule, demonstrating a non-significant 
reduction in IC $(9.8 \%$ vs $16.7 \%, p=0.14)$ and no difference in all-cause mortality $(16.7 \%$ vs $14.3 \%$, $\mathrm{p}=0.78)$.

The similarly-sized INTENSE trial[58] $(n=241)$ randomized ICU patients with intra-abdominal infection requiring emergency surgery to 'pre-emptive' micafungin or placebo. Given prescribing was not based on NCBTs or radiology, current definitions would consider this an antifungal prophylaxis trial. There was no reduction in IC incidence (micafungin $11.1 \%$ vs placebo $8.9 \%$ ). AFT was possibly initiated too late (max. 120-hours post-surgery) given many developed IC early in their admission. No details around source control were presented (e.g. drainage of collections/second laparotomies), which may play a more significant role than early AFT in patients with a surgical abdominal focus.

Both trials suggest early AFT based on risk factors alone does not reduce IC incidence or impact mortality. However, they also illustrate the challenges of powering studies adequately: in the MSG-01 trial, IC incidence in the control group was lower than expected, and the INTENSE trial highlighted the importance of selecting the right at-risk group and timepoint for intervention.

\section{Empirical antifungal therapy in ICU}

Given signs and symptoms of IC are non-specific, overlapping with many other infectious and non-infectious aetiologies, empirical AFT to cover the possibility of fungal infection in the septic ICU patient is common practice. A major factor driving empirical therapy are limitations of conventional culture-based methods. Although gold-standard for diagnosing IC, blood culture (BC) sensitivity is suboptimal ( $75 \%$ in bloodstream infection, $\sim 5-20 \%$ in abdominal candidiasis)[1, 69], sterile site sampling (e.g. abdominal pus) often difficult, and time-to-culturepositivity prolonged (2-3 days)[1].

No survival benefit of early AFT in non-neutropenic ICU patients was demonstrated in a 2016 meta-analysis (>2300 patients from $22 \mathrm{RCTs}$ )[70], although criteria triggering antifungal prescribing in the analyzed studies were very heterogenous. Subsequently, the EMPIRICUS trial[59] ( $n=261)$ randomized ventilated patients with evidence of ICU-acquired sepsis, Candida colonization, and multi-organ failure to empirical micafungin or placebo. No improvement in 28day fungal-free survival was demonstrated (68\% vs $60.2 \%, p=0.18)$, despite significant reduction in proven IC in the micafungin arm ( $3 \%$ vs $12 \%, p=0.008)$. Subgroup analysis suggested a trend towards better survival in those with SOFA score >8 (HR, 1.69 [95\% Cl, 0.96-2.94], p=0.07); Demonstrating survival benefit in ICU patient groups, often with multiple co-morbidities and high 
baseline mortality, requires much larger trials to achieve adequate power. Identifying the subset of ICU patients who could benefit from early AFT remains a key challenge.

Guidelines do not address de-escalation or discontinuation of empirical therapy for suspected infection in the absence of microbiological confirmation. In a post-hoc analysis $(n=647)$ of the observational ARMCAND2 study including patients with suspected(57\%) or proven IC(43\%), deescalation (defined as either switch to azole or antifungal discontinuation by day 5) only occurred in 22\% ( $n=142 ; 96$ switched; 48 stopped), of which half had no microbiological evidence of IC[71]. De-escalation was associated with shorter total AFT duration, with no negative impact on mortality or length of ICU stay despite similar illness severity scores between those who did and did not de-escalate. A smaller observational study had similar findings[72]. Nevertheless, the low proportion switched or stopped highlights barriers to de-escalation in practice. This includes reluctance to modify empirical treatment in unstable patients with uncertain diagnoses, alongside a desire to use a fungicidal, well-tolerated agent to cover the possibility of azole-resistant Candida. Yet, for patients on empirical therapy where the clinical picture suggests low IC-risk and BC are negative, discontinuing AFT appears a reasonable option and could be beneficial in preventing resistance emergence. For those where likelihood of IC is deemed moderate-to-high, non-culturebased diagnostics may have a role in informing decisions.

\section{Role of non-culture-based tests}

Non-culture-based tests (NCBTs) have been developed in attempt to overcome the shortfalls of culture-based fungal diagnostics, given their quick turn-around-time, the potential for earlier IC detection and given they may remain positive for longer while on AFT[1]. They include 1,3- $\beta$ - $d$ glucan (BDG), T2 magnetic resonance Candida assay (T2Candida), Multiplex candida real-time PCR, and the detection of mannan antigen (MAg) and anti-mannan IgG antibodies (Anti-Mn) (Table 2). Potential roles for NCBTs include aiding clinical decision-making to guide; 1) the initiation of pre-emptive AFT; 2) the discontinuation or withholding of empirical AFT; 3) monitoring clinical improvement in patients with IC.

NCBTs have been described as "Bayesian"[69]; i.e. they do not deliver a definitive result, but assess the likelihood of infection. IC prevalence varies between ICUs due to differences in casemix and interventions. With variation in the pre-test likelihood, the negative predictive value (NPV) and PPV of NCBTs changes; in higher-risk patients and settings (e.g. surgical ICU) the PPV will rise and the NPV will decrease, and vice-versa[69]. Hence, as recently outlined in Mycoses Study Group recommendations, NCBTs must be requested and interpreted in the context of the pre-test 
likelihood of IC[73]; they suggest that the clinical value of NCBTs is limited when this figure is less than $10 \%$.

\section{Non-culture-based tests to trigger antifungal initiation}

The ideal NCBT for guiding early antifungal initiation needs a high sensitivity to identify IC, but reasonable specificity to avoid over-prescribing. The most widely used NCBT, BDG, has moderate specificity ( 60-85\%[48-50]), marred by false-positivity which may occur due to haemodialysis, blood product administration, high-burden Candida colonisation, and disturbed GI-mucosa; all common in ICU. This may result in antifungal overuse. Establishing diagnostic cutoff values which optimise test performance in ICU is crucial. BDG specificity improves with consecutive sampling and increasing the 'positive' cut-off value to $\geq 250 \mathrm{pg} / \mathrm{ml}$ (instead of $80 \mathrm{pg} / \mathrm{ml}[74]$ ) which in one study increased specificity to $87 \%$ but reduced sensitivity to $52 \%[75]$. NCBT combinations may also improve specificity; a positive BDG ( $\geq 80 \mathrm{pg} / \mathrm{ml}$ ) alongside a negative PCT (<2 ng/ml) had a 96\% PPV for candidaemia, when distinguishing IC from bacteraemia in one study[76]. Additionally, a highly-positive BDG (>259 pg/ml) alongside a positive CAGTA better distinguished IC from candida colonisation in patients with severe gastrointestinal conditions, compared to either used alone[75]. A prospective Danish study $(n=126)$ in ICU patients at high-risk of IC (particularly IAC) found a combination of T2Candida and BC compared to $\mathrm{MAg}$ and $\mathrm{BC}$, or $\mathrm{BC}$ alone had a the greatest specificity $(64 \% / 53 \% / 29 \%$ respectively), and a sensitivity of $>95 \%$, for diagnosing proven/likely IC[77]. Additionally, a retrospective study assessing NCBTs performance for IAC $(n=48)$ found the sensitivity/specificity for T2Candida was 33\%/93\% and BDG 83\%/67\%; however concordant positive results diagnosed IAC in $100 \%$ of cases, and concordant negative results excluded IAC in $90 \%$ of cases, suggesting combinations would be more useful clinically[78].

To date, few prospective studies have examined the impact of NCBT-driven pre-emptive AFT on outcomes[79]. A small pilot RCT ( $n=64)$ administered pre-emptive anidulafungin to ICU patients with a BDG $\geq 60 \mathrm{pg} / \mathrm{ml}[56]$ during twice-weekly surveillance; while it demonstrated feasibility, enrolment difficulties meant it was not powered to assess a difference in IC or survival. In a subgroup analysis of the EMPIRICUS study, fungal-free survival was not significantly different in those with an elevated BDG who received micafungin versus placebo (BDG > 80 [HR 1.41, 95\% $\mathrm{Cl} 0.85-2.33$ ], $\mathrm{BDG}>200$ [HR 1.51, 95\% Cl 0.47-5.00]), but the trend was in the direction of the micafungin arm[59]. 
Current evidence is not robust enough to support the use of NCBTs alone to trigger AFT. The moderate specificity of BDG hinders its use, but combination with other NCBTs, optimising 'cutoff' values, and directing testing to 'high risk' patients using Candida risk scores, make it a more valuable tool. NCBT-driven pre-emptive therapy using NCBT combinations which maximise test performance (T2Candida plus BDG) alongside culture is the most promising early AFT strategy which needs to be examined robustly in randomised multi-centre clinical trials. Outcomes should include IC, mortality, AFT consumption and cost-effectiveness, so benefits and risks of such a strategy can be holistically assessed. We eagerly await the results of the CandiSep trial (NCT02734550) comparing clinical outcomes of a BDG-driven versus culture-driven approach to AFT prescribing in septic ICU patients.

\section{Non-culture-based tests to aid antifungal discontinuation}

NCBTs with a high NPV may be better used to guide discontinuation or preventing initiation of AFT. A trial $(n=109)$ randomized patients with evidence of infection who fulfilled IC-risk criteria to 14-days' empirical AFT or a NCBT-driven strategy, whereby AFT was stopped if BDG, MAg, Anti$\mathrm{Mn}$, and BC were negative[61]. Unsurprisingly, given comparison was to a 14-day standard, AFT duration was shorter in the NCBT arm, but importantly there was no deleterious impact on mortality or development of IC. A further study $(n=85)$ prescribed empirical AFT to patients with risk factors and signs of infection[60]; based on negative BC and serial negative BDGs, AFT was safely discontinued in $21 / 85$ by day 4 and none developed candidaemia upon follow-up. Other retrospective studies demonstrated similar findings[80, 81]. An ICU study assessing utility of BDG for therapeutic decision-making found that although introduced to target patients 'high-risk' of IC, in practice only $26 \%$ of patients in whom it was used were in this category[82]. Results influenced AFT prescribing in over half of cases, deemed appropriate in three quarters and inappropriate in a quarter of cases (AFT continued/started with no subsequent evidence of IFI). Thus paradoxically, in real-world deployment of ICU BDG testing, any reductions in antifungal consumption gained through earlier stopping of inappropriate therapy based on the test's good NPV may be outweighed by an excess in prescribing due to the test's poor PPV when used in an unselected population. Studies so far have been too small to assess the safety and clinical effectiveness of NCBT-driven AFT discontinuation algorithms. Multi-site studies comprising a range of low-to-high IC prevalence settings are needed for results to be generalisable.

In summary, whilst NCBT results interpreted in context remain a useful adjunct in stewardship, to date there is insufficient evidence to support antifungal discontinuation based on negative NCBT[48, 83]. The A-STOP trial (ISRCTN43895480), a large multi-site (35 hospital) UK 
diagnostic accuracy study prospectively assessing which NCBT (or combination thereof) can best rule-out IC and facilitate AFT discontinuation in ICU patients, holds promise of delivering on this.

Non-Culture-Based Tests for Monitoring

Clinical response markers for monitoring and prognostication in IC are lacking. BC clearance of fungi is often used as a proxy for treatment effectiveness, however this is less-than-ideal given their suboptimal sensitivity, particularly in deep-seated candidiasis.

Studies in ICU[77] and mixed ward/ICUs[84], found T2Candida remained detectable for longer than $B C$ in candidaemia; in the latter study $7.5 \%(4 / 31)$ had a positive surveillance $B C$, yet $41.9 \%$ (13/31) had a positive surveillance T2Candida. Hence, time-to-negative result with T2Candida was significantly longer, perhaps unsurprising given T2Candida also detects non-viable Candida cells. To assess its clinical relevance, larger studies correlating persistent T2Candida positivity with clinical outcomes are needed. While studies have examined BDG kinetics for monitoring treatment response in IC[85-87], few have done so in an ICU-specific population[88, 89]. In heterogenous patient groups, serial BDG decline has been associated with successful therapy, with a slower decrease in patients with deep-seated candidiasis[85, 86], and persistently negative BDGs in candidaemic patients are associated with a lower 30-day mortality[90, 91]. However, in ICU patients with intra-abdominal candidiasis[88] and candididaemia[89] BDG was slow to clear from circulation and remained positive beyond clinical resolution of infection. Hence, while the trajectory of decline, or persistent negativity may have some monitoring use, there is little evidence to support that transition from a positive to negative BDG is valuable in assessing treatment response and currently no evidence that it can be used to guide AFT duration. 


\section{Challenge 3: Choosing the Optimal Antifungal Drug for the ICU Patient}

The antifungal armamentarium is limited, with just four classes of drugs available for treatment of IC; azoles (fluconazole, voriconazole); echinocandins (caspofungin, anidulafungin, micafungin); polyenes (amphotericin B); and the pyrimidine analogue, flucytosine. Drug development is progressing, with several new agents undergoing trials (e.g. Ibrexafungerp, fosmanogepix, rezafungin)[92].

Several guidelines aid the appropriate selection of an antifungal[93, 94]. Echinocandins are recommended first-line treatment of proven[93, 94] and suspected[93] IC in non-neutropenic critically ill adults, due to their broader-spectrum compared to fluconazole, fungicidal activity, excellent tolerability and minimal drug interactions. In the only comparative RCT, anidulafungin was found to be non-inferior to fluconazole for the treatment of IC (global response $73.2 \%$ versus $61.1 \%, 95 \% \mathrm{Cl},-1.1$ to 25.3 )[95]. A post-hoc subgroup analysis in ICU patients demonstrated significantly better response rates for those receiving an echinocandin $(70.8 \%$ versus $54.1 \%$, $\mathrm{p}=0.03)$, although this did not translate to a reduction in 28 -day mortality $(20.2 \%$ versus $24.3 \% P$ $=0.57$ )[96]. Other observational studies comparing mortality between those initiated on fluconazole or echinocandins, showed either no difference[97-99], or favoured echinocandins[100, 101]. However, adjusting for the multiple confounders influencing outcome in ICU in non-randomised studies is difficult. A recent large RCT failed to demonstrate non-inferiority of the newest triazole, isavuconazole, when compared to caspofungin for IC (end-of-IV-therapy treatment response, $60.3 \%$ versus $70.1 \%, 95 \% \mathrm{Cl}-19.9--1.8)$, consistent regardless of illness severity[102]. There is no evidence to suggest a difference in efficacy or mortality with Amphotericin B compared to azoles and echinocandins[103]. Given the higher cost and association with greater toxicity, amphotericin B in IC treatment is usually reserved for situations with no suitable alternatives eg. MDR Candida, for drug-penetration.

In candidaemia, de-escalation from echinocandins to fluconazole for azole-susceptible isolates, when repeat $\mathrm{BCs}$ are negative and the patient is clinically stable is recommended within 5-7 days in IDSA[93], and at 10-days in ESCMID guidelines[94]. A number of studies, (albeit not RCTs) have demonstrated the safety of this approach at day 5 in proven IC[71, 100,104] with no impact on clinical outcomes. The ESGCIP taskforce recently recommended considering de-escalation at day 5, dependent upon clinical response[105]. 


\section{Antifungal drugs in special ICU populations}

Alongside guidelines, patient-specific factors need to be considered when choosing the most appropriate drug, dose and duration for different clinical scenarios[106], summarised in table 4.

Acute kidney injury $(\mathrm{AKI})$ is common in ICU, sometimes requiring continuous renal replacement therapy (CRRT) which can significantly affect antifungal PK/PD. The kidneys excrete $60-80 \%$ of fluconazole unchanged[107]; dose reduction in AKI is thus required due to delayed elimination. Conversely, high elimination is seen with CRRT due to low protein-binding and high water solubility, therefore increased fluconazole doses are advised[107]. For voriconazole, no renal or CRRT dose adjustment is required but frequent TDM is needed. Given the voriconazole IV solvent vehicle can accumulate in moderate-to-severe renal impairment, oral over IV therapy is recommended[107]. Amphotericin B, particularly the deoxycholate formulation, can be associated with nephrotoxicity and should be avoided in renal impairment if suitable alternatives are available. CRRT dose adjustment is not required. Echinocandins are highly protein-bound with minimal renal excretion, therefore no dose adjustment is required in renal failure, and CRRT has no clinically significant effect on drug removal[108], making them an optimal choice.

Chronic and acute liver failure is frequently seen in ICU patients. Drug-induced liver injury (DILI) is a risk with all azoles, therefore caution is required in pre-existing moderate or severe liver disease and alternatives considered. No dose adjustment is required for amphotericin [109]. Anidulafungin is the only echinocandin eliminated through extrahepatic metabolism[110] and therefore often the preferred agent in hepatic impairment.

ECMO is increasingly used for cardiorespiratory support in ICU; altered antifungal PK/PD may occur due to drug sequestration, increased volume of distribution, and drug clearance changes while on the ECMO circuit, but data are scarce[111]. Micafungin extraction by ECMO was demonstrated in an ex-vivo study[112], however there are conflicting data with caspofungin[113, 114], the latter study demonstrating adequate levels at usual doses. Satisfactory liposomal amphotericin B levels at standard dosing on ECMO are reported[115], while others administering higher doses $(10 \mathrm{mg} / \mathrm{kg} / \mathrm{day})$ found a $\sim 50 \%$ reduction in $C_{\max }[116]$. Due to increased volume distribution, larger fluconazole loading doses were required in children, however adult data are lacking[111, 117]. Voriconazole sequestration is reported, although the degree of sequestration changes with time, possibly due to saturation of ECMO circuit binding sites[114]. Hence frequent azole TDM is crucial yet rarely available in real-time, highlighting a pressing need for development of point-of-care antifungal TDM in ICU patients. Given expanding ICU ECMO use and its 
association with higher IFI risk, further antifungal PK/PD studies are needed as current data is insufficient to adequately inform antifungal ECMO guidelines.

\section{CONCLUSION}

The diagnosis and management of IC poses many challenges in critical care; numerous unanswered questions remain as research priorities (Table 5). Improved identification of at-risk patients and the widening spectrum of diagnostics and therapeutics available for IC are promising. Personalized approaches to drug dosing and monitoring treatment response are needed. The key knowledge gap remaining is how tools such as risk scores and NCBTs can best be implemented in ICU practice to optimise clinical outcomes whilst exercising antifungal stewardship. 


\section{REFERENCES}

1. Clancy CJ, Nguyen MH (2013) Finding the missing $50 \%$ of invasive candidiasis: How nonculture diagnostics will improve understanding of disease spectrum and transform patient care. Clin Infect Dis 56:1284-1292. https://doi.org/10.1093/cid/cit006

2. Poissy J, Damonti L, Bignon A, et al (2020) Risk factors for candidemia: A prospective matched case-control study. Crit Care 24:. https://doi.org/10.1186/s13054-020-2766-1

3. Delaloye J, Calandra T (2014) Invasive candidiasis as a cause of sepsis in the critically ill patient. Virulence 5:161-9. https://doi.org/10.4161/viru.26187

4. Kett DH, Azoulay E, Echeverria PM, Vincent JL (2011) Candida bloodstream infections in intensive care units: Analysis of the extended prevalence of infection in intensive care unit study. Crit Care Med 39:665-670. https://doi.org/10.1097/CCM.0b013e318206c1ca

5. Paiva JA, Pereira JM, Tabah A, et al (2016) Characteristics and risk factors for 28-day mortality of hospital acquired fungemias in ICUs: Data from the EUROBACT study. Crit Care 20:53. https://doi.org/10.1186/s13054-016-1229-1

6. Bassetti M, Giacobbe DR, Vena A, et al (2019) Incidence and outcome of invasive candidiasis in intensive care units (ICUs) in Europe: results of the EUCANDICU project. Crit Care 23:219. https://doi.org/10.1186/s13054-019-2497-3

7. Koehler P, Stecher M, Cornely OA, et al (2019) Morbidity and mortality of candidaemia in Europe: an epidemiologic meta-analysis. Clin Microbiol Infect 25:1200-1212. https://doi.org/10.1016/j.cmi.2019.04.024

8. Bassetti M, Righi E, Ansaldi F, et al (2015) A multicenter multinational study of abdominal candidiasis: epidemiology, outcomes and predictors of mortality. Intensive Care Med 41:1601-1610. https://doi.org/10.1007/s00134-015-3866-2

9. Kullberg BJ, Arendrup MC (2015) Invasive candidiasis. N. Engl. J. Med. 373:1445-1456

10. Spellberg BJ, Filler SG, Edwards JE (2006) Current Treatment Strategies for Disseminated Candidiasis. Clin Infect Dis 42:244-251. https://doi.org/10.1086/499057

11. Kumar V, Cheng S-C, Johnson MD, et al (2014) Immunochip SNP array identifies novel genetic variants conferring susceptibility to candidaemia. Nat Commun 5:4675. https://doi.org/10.1038/ncomms5675

12. Vincent J-L, Lefrant J-Y, Kotfis K, et al (2018) Comparison of European ICU patients in 2012 (ICON) versus 2002 (SOAP). Intensive Care Med 44:337-344.

https://doi.org/10.1007/s00134-017-5043-2

13. Karagiannidis C, Brodie D, Strassmann S, et al (2016) Extracorporeal membrane oxygenation: evolving epidemiology and mortality. Intensive Care Med 42:889-896. https://doi.org/10.1007/s00134-016-4273-z

14. Bell T, O'Grady NP (2017) Prevention of Central Line-Associated Bloodstream Infections. Infect. Dis. Clin. North Am. 31:551-559

15. Vincent JL, Sakr Y, Singer M, et al (2020) Prevalence and Outcomes of Infection among Patients in Intensive Care Units in 2017. JAMA - J Am Med Assoc 323:1478-1487. https://doi.org/10.1001/jama.2020.2717

16. Tortorano AM, Dho G, Prigitano A, et al (2012) Invasive fungal infections in the intensive 
care unit: A multicentre, prospective, observational study in Italy (2006-2008). Mycoses 55:73-79. https://doi.org/10.1111/j.1439-0507.2011.02044.x

17. Montagna MT, Caggiano G, Lovero G, et al (2013) Epidemiology of invasive fungal infections in the intensive care unit: Results of a multicenter Italian survey (AURORA Project). Infection 41:645-653

18. Baldesi O, Bailly S, Ruckly S, et al (2017) ICU-acquired candidaemia in France: Epidemiology and temporal trends, 2004-2013 - A study from the REA-RAISIN network. J Infect 75:59-67. https://doi.org/10.1016/j.jinf.2017.03.011

19. Lamoth F, Lockhart SR, Berkow EL, Calandra T (2018) Changes in the epidemiological landscape of invasive candidiasis. J Antimicrob Chemother 73:i4-i13.

https://doi.org/10.1093/jac/dkx444

20. Chakrabarti A, Sood P, Rudramurthy SM, et al (2015) Incidence, characteristics and outcome of ICU-acquired candidemia in India. Intensive Care Med 41:285-295. https://doi.org/10.1007/s00134-014-3603-2

21. Nucci M, Queiroz-Telles F, Alvarado-Matute T, et al (2013) Epidemiology of Candidemia in Latin America: A Laboratory-Based Survey. PLoS One 8:e59373.

https://doi.org/10.1371/journal.pone.0059373

22. Lockhart SR, lqbal N, Cleveland AA, et al (2012) Species identification and antifungal susceptibility testing of Candida bloodstream isolates from population-based surveillance studies in two U.S. cities from 2008 to 2011. J Clin Microbiol 50:3435-3442.

https://doi.org/10.1128/JCM.01283-12

23. Pfaller MA, Diekema DJ, Turnidge JD, et al (2019) Twenty Years of the SENTRY Antifungal Surveillance Program: Results for Candida Species From 1997-2016. Open forum Infect Dis 6:S79-S94. https://doi.org/10.1093/ofid/ofy358

24. Alexander BD, Johnson MD, Pfeiffer CD, et al (2013) Increasing echinocandin resistance in candida glabrata: Clinical failure correlates with presence of FKS mutations and elevated minimum inhibitory concentrations. Clin Infect Dis 56:1724-1732. https://doi.org/10.1093/cid/cit136

25. Pfaller MA, Castanheira M, Lockhart SR, et al (2012) Frequency of decreased susceptibility and resistance to echinocandins among fluconazole-resistant bloodstream isolates of Candida glabrata. J Clin Microbiol 50:1199-1203.

https://doi.org/10.1128/JCM.06112-11

26. Vallabhaneni S, Cleveland AA, Farley MM, et al (2015) Epidemiology and risk factors for echinocandin nonsusceptible Candida glabrata bloodstream infections: Data from a large multisite population-based candidemia surveillance program, 2008-2014. Open Forum Infect Dis 2:. https://doi.org/10.1093/ofid/ofv163

27. Pham CD, lqbal N, Bolden CB, et al (2014) Role of FKS mutations in Candida glabrata: MIC values, echinocandin resistance, and multidrug resistance. Antimicrob Agents Chemother 58:4690-4696. https://doi.org/10.1128/AAC.03255-14

28. Cortegiani A, Misseri G, Chowdhary A (2019) What's new on emerging resistant Candida species. Intensive Care Med. 45:512-515

29. Van Schalkwyk E, Mpembe RS, Thomas J, et al (2019) Epidemiologic shift in Candidemia driven by Candida auris, South Africa, 2016-2017. Emerg Infect Dis 
25:1698-1707. https://doi.org/10.3201/eid2509.190040

30. Ostrowsky B, Greenko J, Adams E, et al (2020) Candida auris Isolates Resistant to Three Classes of Antifungal Medications - New York, 2019. MMWR Morb Mortal Wkly Rep 69:6-9. https://doi.org/10.15585/mmwr.mm6901a2

31. Eggimann P, Pittet D (2014) Candida colonization index and subsequent infection in critically ill surgical patients: 20 years later. Intensive Care Med 40:1429-1448. https://doi.org/10.1007/s00134-014-3355-z

32. Lortholary O, Desnos-Ollivier M, Sitbon K, et al (2011) Recent exposure to caspofungin or fluconazole influences the epidemiology of candidemia: A prospective multicenter study involving 2,441 patients. Antimicrob Agents Chemother 55:532-538. https://doi.org/10.1128/AAC.01128-10

33. Jensen $\mathrm{RH}$, Johansen HK, Søes LM, et al (2016) Posttreatment antifungal resistance among colonizing Candida isolates in candidemia patients: Results from a systematic multicenter study. Antimicrob Agents Chemother 60:1500-1508.

https://doi.org/10.1128/AAC.01763-15

34. Healey KR, Nagasaki Y, Zimmerman M, et al (2017) The gastrointestinal tract is a major source of echinocandin drug resistance in a murine model of Candida glabrata colonization and systemic dissemination. Antimicrob Agents Chemother 61: https://doi.org/10.1128/AAC.01412-17

35. Thomaz DY, De Almeida JN, Lima GME, et al (2018) An azole-resistant candida parapsilosis outbreak: Clonal persistence in the intensive care unit of a Brazilian teaching hospital. Front Microbiol 9:. https://doi.org/10.3389/fmicb.2018.02997

36. Goemaere B, Lagrou K, Spriet I, et al (2018) Clonal spread of candida glabrata bloodstream isolates and fluconazole resistance affected by prolonged exposure: A 12year single-center study in Belgium. Antimicrob Agents Chemother 62:. https://doi.org/10.1128/AAC.00591-18

37. Schelenz S, Hagen F, Rhodes JL, et al (2016) First hospital outbreak of the globally emerging Candida auris in a European hospital. Antimicrob Resist Infect Control 5:35. https://doi.org/10.1186/s13756-016-0132-5

38. Ku TSN, Walraven CJ, Lee SA (2018) Candida auris: Disinfectants and implications for infection control. Front. Microbiol. 9

39. Eyre DW, Sheppard AE, Madder H, et al (2018) A Candida auris Outbreak and Its Control in an Intensive Care Setting. N Engl J Med 379:1322-1331. https://doi.org/10.1056/NEJMoa1714373

40. Centers for Disease Control and Prevention (2020) Infection Prevention and Control for Candida auris. In: Fungal Dis. https://www.cdc.gov/fungal/candida-auris/c-auris-infectioncontrol.html

41. Public Health England (2017) Candida auris: laboratory investigation, management and infection prevention and control. In: Public Heal. Engl.

https://www.gov.uk/government/publications/candida-auris-laboratory-investigationmanagement-and-infection-prevention-and-control

42. Caceres DH, Forsberg K, Welsh RM, et al (2019) Candida auris: A Review of Recommendations for Detection and Control in Healthcare Settings. J Fungi 5:111. 
https://doi.org/10.3390/jof5040111

43. Kollef M, Micek S, Hampton N, et al (2012) Septic Shock Attributed to Candida Infection: Importance of Empiric Therapy and Source Control. Clin Infect Dis 54:1739-1746. https://doi.org/10.1093/cid/cis305

44. Azoulay E, Dupont H, Tabah A, et al (2012) Systemic antifungal therapy in critically ill patients without invasive fungal infection*. Crit Care Med 40:813-22. https://doi.org/10.1097/CCM.0b013e318236f297

45. Pittet D, Monod M, Suter PM, et al (1994) Candida colonization and subsequent infections in critically ill surgical patients. Ann Surg 220:751-758.

https://doi.org/10.1097/00000658-199412000-00008

46. León C, Ruiz-Santana S, Saavedra P, et al (2006) A bedside scoring system ("Candida score") for early antifungal treatment in nonneutropenic critically ill patients with Candida colonization. Crit Care Med 34:730-737.

https://doi.org/10.1097/01.CCM.0000202208.37364.7D

47. Ostrosky-Zeichner L, Pappas PG, Shoham S, et al (2011) Improvement of a clinical prediction rule for clinical trials on prophylaxis for invasive candidiasis in the intensive care unit. Mycoses 54:46-51. https://doi.org/10.1111/j.1439-0507.2009.01756.x

48. Haydour Q, Hage CA, Carmona EM, et al (2019) Diagnosis of fungal infections a systematic review and meta-analysis supporting American thoracic society practice guideline. Ann. Am. Thorac. Soc. 16:1179-1188

49. Karageorgopoulos DE, Vouloumanou EK, Ntziora F, et al (2011) -D-Glucan Assay for the Diagnosis of Invasive Fungal Infections: A Meta-analysis. Clin Infect Dis 52:750-770. https://doi.org/10.1093/cid/ciq206

50. He S, Hang JP, Zhang L, et al (2015) A systematic review and meta-analysis of diagnostic accuracy of serum 1,3- $\beta$-d-glucan for invasive fungal infection: Focus on cutoff levels. J. Microbiol. Immunol. Infect. 48:351-361

51. Mikulska M, Calandra T, Sanguinetti M, et al (2010) The use of mannan antigen and antimannan antibodies in the diagnosis of invasive candidiasis: Recommendations from the Third European Conference on Infections in Leukemia. Crit Care 14:. https://doi.org/10.1186/cc9365

52. Wei S, Wu T, Wu Y, et al (2019) Diagnostic accuracy of Candida albicans germ tube antibody for invasive candidiasis: systematic review and meta-analysis. Diagn Microbiol Infect Dis 93:339-345. https://doi.org/10.1016/j.diagmicrobio.2018.10.017

53. Chang SS, Hsieh WH, Liu TS, et al (2013) Multiplex PCR System for Rapid Detection of Pathogens in Patients with Presumed Sepsis - A Systemic Review and Meta-Analysis. PLoS One 8:. https://doi.org/10.1371/journal.pone.0062323

54. Avni T, Leibovici L, Paul M (2011) PCR diagnosis of invasive candidiasis: Systematic review and meta-analysis. J Clin Microbiol 49:665-670.

https://doi.org/10.1128/JCM.01602-10

55. Tang DL, Chen X, Zhu CG, et al (2019) Pooled analysis of T2 Candida for rapid diagnosis of candidiasis. BMC Infect Dis 19:798. https://doi.org/10.1186/s12879-0194419-z 
56. Hanson KE, Pfeiffer CD, Lease ED, et al (2012) $\beta$-D-glucan surveillance with preemptive anidulafungin for invasive candidiasis in intensive care unit patients: a randomized pilot study. PLoS One 7:e42282. https://doi.org/10.1371/journal.pone.0042282

57. Ostrosky-Zeichner L, Shoham S, Vazquez J, et al (2014) MSG-01: A randomized, double-blind, placebo-controlled trial of caspofungin prophylaxis followed by preemptive therapy for invasive candidiasis in high-risk adults in the critical care setting. Clin Infect Dis 58:1219-26. https://doi.org/10.1093/cid/ciu074

58. Knitsch W, Vincent J-L, Utzolino S, et al (2015) A randomized, placebo-controlled trial of preemptive antifungal therapy for the prevention of invasive candidiasis following gastrointestinal surgery for intra-abdominal infections. Clin Infect Dis 61:1671-8. https://doi.org/10.1093/cid/civ707

59. Timsit JF, Azoulay E, Schwebel C, et al (2016) Empirical micafungin treatment and survival without invasive fungal infection in adults with icu-acquired sepsis, candida colonization, and multiple organ failure the empiricus randomized clinical trial. JAMA - J Am Med Assoc 316:1555-1564. https://doi.org/10.1001/jama.2016.14655

60. Nucci M, Nouér SA, Esteves $P$, et al (2016) Discontinuation of empirical antifungal therapy in ICU patients using 1,3- $\beta$-d-glucan. J Antimicrob Chemother 71:2628-2633. https://doi.org/10.1093/jac/dkw188

61. Rouzé A, Loridant S, Poissy J, et al (2017) Biomarker-based strategy for early discontinuation of empirical antifungal treatment in critically ill patients: a randomized controlled trial. Intensive Care Med 43:1668-1677. https://doi.org/10.1007/s00134-0174932-8

62. Pappas PG, Lionakis MS, Arendrup MC, et al (2018) Invasive candidiasis. Nat Rev Dis Prim 4:18026. https://doi.org/10.1038/nrdp.2018.26

63. Eggimann P, Bille J, Marchetti O (2011) Diagnosis of invasive candidiasis in the ICU. Ann Intensive Care 1:37. https://doi.org/10.1186/2110-5820-1-37

64. Garbino J, Lew DP, Jacques-A. Romand, et al (2002) Prevention of severe Candida infections in nonneutropenic, high-risk, critically ill patients: A randomized, double-blind, placebo-controlled trial in patients treated by selective digestive decontamination. Intensive Care Med 28:1708-1717. https://doi.org/10.1007/s00134-002-1540-y

65. Piarroux R, Grenouillet F, Balvay P, et al (2004) Assessment of preemptive treatment to prevent severe candidiasis in critically ill surgical patients. Crit Care Med 32:2443-9. https://doi.org/10.1097/01.ccm.0000147726.62304.7f

66. Senn L, Eggimann P, Ksontini R, et al (2009) Caspofungin for prevention of intraabdominal candidiasis in high-risk surgical patients. Intensive Care Med 35:903-908. https://doi.org/10.1007/s00134-009-1405-8

67. Ferreira D, Grenouillet F, Blasco G, et al (2015) Outcomes associated with routine systemic antifungal therapy in critically ill patients with Candida colonization. Intensive Care Med 41:1077-1088. https://doi.org/10.1007/s00134-015-3791-4

68. Harrison D, Muskett H, Harvey S, et al (2013) Development and validation of a risk model for identification of non-neutropenic, critically-ill, adult patients at high risk of invasive Candida infection. Health Technol Assess (Rockv) 17:1-30.

https://doi.org/10.3310/hta17030 
69. Clancy CJ, Nguyen MH (2018) Non-culture diagnostics for invasive candidiasis: Promise and unintended consequences. J. Fungi 4:27

70. Cortegiani A, Russotto V, Maggiore A, et al (2016) Antifungal agents for preventing fungal infections in non-neutropenic critically ill patients. Cochrane Database Syst. Rev. 2016

71. Bailly S, Leroy O, Montravers P, et al (2015) Antifungal de-escalation was not associated with adverse outcome in critically ill patients treated for invasive candidiasis: post hoc analyses of the AmarCAND2 study data. Intensive Care Med 41:1931-1940. https://doi.org/10.1007/s00134-015-4053-1

72. Jaffal K, Poissy J, Rouze A, et al (2018) De-escalation of antifungal treatment in critically ill patients with suspected invasive Candida infection: incidence, associated factors, and safety. Ann Intensive Care 8:49. https://doi.org/10.1186/s13613-018-0392-8

73. Johnson MD, Lewis RE, Dodds Ashley ES, et al (2020) Core Recommendations for Antifungal Stewardship: A Statement of the Mycoses Study Group Education and Research Consortium. J Infect Dis 222:S175-S198. https://doi.org/10.1093/infdis/jiaa394

74. Martin-Loeches I, Antonelli M, Cuenca-Estrella M, et al (2019) ESICM/ESCMID task force on practical management of invasive candidiasis in critically ill patients. Intensive Care Med 45:789-805. https://doi.org/10.1007/s00134-019-05599-w

75. León C, Ruiz-Santana S, Saavedra P, et al (2012) Value of $\beta$-D-glucan and Candida albicans germ tube antibody for discriminating between Candida colonization and invasive candidiasis in patients with severe abdominal conditions. Intensive Care Med 38:1315-1325. https://doi.org/10.1007/s00134-012-2616-y

76. Giacobbe DR, Mikulska M, Tumbarello $M$, et al (2017) Combined use of serum (1,3)- $\beta-d-$ glucan and procalcitonin for the early differential diagnosis between candidaemia and bacteraemia in intensive care units. Crit Care 21:176. https://doi.org/10.1186/s13054017-1763-5

77. Arendrup MC, Andersen JS, Holten MK, et al (2019) Diagnostic Performance of T2Candida Among ICU Patients With Risk Factors for Invasive Candidiasis. Open forum Infect Dis 6:ofz136. https://doi.org/10.1093/ofid/ofz136

78. Lamoth F, Clancy CJ, Tissot F, et al (2020) Performance of the T2Candida panel for the diagnosis of intra-abdominal candidiasis. Open Forum Infect Dis 7:.

https://doi.org/10.1093/ofid/ofaa075

79. Rouze A, Poissy J, Sendid B, Nseir S (2018) Biomarkers in early treatment of invasive candidiasis. Hosp Pract 46:239-242. https://doi.org/10.1080/21548331.2018.1516105

80. Gill CM, Kenney RM, Hencken L, et al (2019) T2 Candida versus beta-D-glucan to facilitate antifungal discontinuation in the intensive care unit. Diagn Microbiol Infect Dis 95:162-165. https://doi.org/10.1016/J.DIAGMICROBIO.2019.04.016

81. Rautemaa-Richardson R, Rautemaa V, Al-Wathiqi F, et al (2018) Impact of a diagnosticsdriven antifungal stewardship programme in a UK tertiary referral teaching hospital. $\mathrm{J}$ Antimicrob Chemother 73:3488-3495. https://doi.org/10.1093/jac/dky360

82. Kritikos A, Poissy J, Poissy J, et al (2020) Impact of the beta-glucan test on management of intensive care unit patients at risk for invasive candidiasis. J Clin Microbiol 58:.

https://doi.org/10.1128/JCM.01996-19 
83. Hage CA, Carmona EM, Epelbaum O, et al (2019) Microbiological laboratory testing in the diagnosis of fungal infections in pulmonary and critical care practice: An official American thoracic society clinical practice guideline. Am. J. Respir. Crit. Care Med. 200:535-550

84. Mylonakis E, Zacharioudakis IM, Clancy CJ, et al (2018) Efficacy of T2 Magnetic Resonance Assay in Monitoring Candidemia after Initiation of Antifungal Therapy: the Serial Therapeutic and Antifungal Monitoring Protocol (STAMP) Trial. J Clin Microbiol 56:e01756-17. https://doi.org/10.1128/JCM.01756-17

85. Jaijakul S, Vazquez JA, Swanson RN, Ostrosky-Zeichner L (2012) (1,3)- -D-Glucan as a Prognostic Marker of Treatment Response in Invasive Candidiasis. Clin Infect Dis 55:521-526. https://doi.org/10.1093/cid/cis456

86. Angebault C, Lanternier F, Dalle F, et al (2016) Prospective Evaluation of Serum $\beta$ Glucan Testing in Patients With Probable or Proven Fungal Diseases. Open Forum Infect Dis 3:ofw128. https://doi.org/10.1093/ofid/ofw128

87. Koo S, Baden LR, Marty FM (2012) Post-diagnostic kinetics of the $(1 \rightarrow 3)$ - $\beta$-d-glucan assay in invasive aspergillosis, invasive candidiasis and Pneumocystis jirovecii pneumonia. Clin Microbiol Infect 18:E122-7. https://doi.org/10.1111/j.14690691.2012.03777.x

88. Tissot F, Lamoth F, Hauser PM, et al (2013) $\beta$-Glucan Antigenemia Anticipates Diagnosis of Blood Culture-Negative Intraabdominal Candidiasis. Am J Respir Crit Care Med 188:1100-1109. https://doi.org/10.1164/rccm.201211-20690C

89. Poissy J, Sendid B, Damiens S, et al (2014) Presence of Candida cell wall derived polysaccharides in the sera of intensive care unit patients: Relation with candidaemia and Candida colonisation. Crit Care 18:R135. https://doi.org/10.1186/cc13953

90. Giacobbe DR, Berruti M, Mikulska M (2019) Prognostic impact of negative serum $(1,3)-\beta$ D-glucan in patients with candidemia. Clin Infect Dis. https://doi.org/10.1093/cid/ciz1051

91. Agnelli C, Bouza E, Martínez-Jiménez MDC, et al (2020) Clinical Relevance and Prognostic Value of Persistently Negative $(1,3)-\beta-D-G l u c a n$ in Adults with Candidemia: A 5-year Experience in a Tertiary Hospital. Clin Infect Dis 70:1925-1932. https://doi.org/10.1093/cid/ciz555

92. Gintjee TJ, Donnelley MA, Thompson GR (2020) Aspiring Antifungals: Review of Current Antifungal Pipeline Developments. J Fungi 6:28. https://doi.org/10.3390/jof6010028

93. Pappas PG, Kauffman CA, Andes DR, et al (2015) Clinical Practice Guideline for the Management of Candidiasis: 2016 Update by the Infectious Diseases Society of America. Clin. Infect. Dis. 62:e1-e50

94. Cornely OA, Bassetti M, Calandra T, et al (2012) ESCMID guideline for the diagnosis and management of Candida diseases 2012: non-neutropenic adult patients. Clin Microbiol Infect 18:19-37. https://doi.org/10.1111/1469-0691.12039

95. Reboli AC, Rotstein C, Pappas PG, et al (2007) Anidulafungin versus Fluconazole for Invasive Candidiasis. N Engl J Med 356:2472-2482.

https://doi.org/10.1056/NEJMoa066906

96. Kett DH, Shorr AF, Reboli AC, et al (2011) Anidulafungin compared with fluconazole in severely ill patients with candidemia and other forms of invasive candidiasis: Support for 
the 2009 IDSA treatment guidelines for candidiasis. Crit Care 15:R253. https://doi.org/10.1186/cc10514

97. López-Cortés LE, Almirante B, Cuenca-Estrella M, et al (2016) Empirical and targeted therapy of candidemia with fluconazole versus echinocandins: a propensity scorederived analysis of a population-based, multicentre prospective cohort. Clin Microbiol Infect 22:733.e1-733.e8. https://doi.org/10.1016/j.cmi.2016.05.008

98. Murri R, Scoppettuolo G, Ventura G, et al (2016) Initial antifungal strategy does not correlate with mortality in patients with candidemia. Eur J Clin Microbiol Infect Dis 35:187-193. https://doi.org/10.1007/s10096-015-2527-2

99. Bassetti M, Righi E, Ansaldi F, et al (2014) A multicenter study of septic shock due to candidemia: Outcomes and predictors of mortality. Intensive Care Med 40:839-845. https://doi.org/10.1007/s00134-014-3310-z

100. Garnacho-Montero J, Díaz-Martín A, Cantón-Bulnes L, et al (2018) Initial antifungal strategy reduces mortality in critically ill patients with candidemia: A propensity scoreadjusted analysis of a multicenter study. Crit Care Med 46:384-393. https://doi.org/10.1097/CCM.0000000000002867

101. Colombo AL, Guimarães T, Sukienik T, et al (2014) Prognostic factors and historical trends in the epidemiology of candidemia in critically ill patients: an analysis of five multicenter studies sequentially conducted over a 9-year period. Intensive Care Med 40:1489-1498. https://doi.org/10.1007/s00134-014-3400-y

102. Kullberg BJ, Viscoli C, Pappas PG, et al (2019) Isavuconazole Versus Caspofungin in the Treatment of Candidemia and Other Invasive Candida Infections: The ACTIVE Trial. Clin Infect Dis 68:1981-1989. https://doi.org/10.1093/cid/ciy827

103. Keane S, Geoghegan P, Povoa P, et al (2018) Systematic review on the first line treatment of amphotericin $B$ in critically ill adults with candidemia or invasive candidiasis. Expert Rev. Anti. Infect. Ther. 16:839-847

104. Vazquez J, Reboli AC, Pappas PG, et al (2014) Evaluation of an early step-down strategy from intravenous anidulafungin to oral azole therapy for the treatment of candidemia and other forms of invasive candidiasis: Results from an open-label trial. BMC Infect Dis 14:. https://doi.org/10.1186/1471-2334-14-97

105. Tabah A, Bassetti M, Kollef MH, et al (2020) Antimicrobial de-escalation in critically ill patients: a position statement from a task force of the European Society of Intensive Care Medicine (ESICM) and European Society of Clinical Microbiology and Infectious Diseases (ESCMID) Critically III Patients Study Group (ESGCIP). Intensive Care Med 46:245-265. https://doi.org/10.1007/s00134-019-05866-w

106. Chatelon J, Cortegiani A, Hammad E, et al (2019) Choosing the Right Antifungal Agent in ICU Patients. Adv. Ther. 36:3308-3320

107. Bellmann R, Smuszkiewicz P (2017) Pharmacokinetics of antifungal drugs: practical implications for optimized treatment of patients. Infection 45:737-779

108. González de Molina FJ, Martínez-Alberici M de LÁ, Ferrer R (2014) Treatment with echinocandins during continuous renal replacement therapy. Crit. Care 18:218

109. Tverdek FP, Kofteridis D, Kontoyiannis DP (2016) Antifungal agents and liver toxicity: a complex interaction. Expert Rev. Anti. Infect. Ther. 14:765-776 
110. Aguilar G, Azanza JR, Carbonell JA, et al (2014) Anidulafungin dosing in critically ill patients with continuous venovenous haemodiafiltration. J Antimicrob Chemother 69:1620-3. https://doi.org/10.1093/jac/dkt542

111. Sherwin J, Heath T, Watt K (2016) Pharmacokinetics and Dosing of Anti-infective Drugs in Patients on Extracorporeal Membrane Oxygenation: A Review of the Current Literature. Clin. Ther. 38:1976-1994

112. Watt KM, Cohen-Wolkowiez M, Williams DC, et al (2017) Antifungal Extraction by the Extracorporeal Membrane Oxygenation Circuit. J Extra Corpor Technol 49:150-159

113. Ruiz S, Papy E, Da Silva D, et al (2009) Potential voriconazole and caspofungin sequestration during extracorporeal membrane oxygenation. Intensive Care Med. 35:183-184

114. Spriet I, Annaert P, Meersseman P, et al (2009) Pharmacokinetics of caspofungin and voriconazole in critically ill patients during extracorporeal membrane oxygenation. $\mathrm{J}$ Antimicrob Chemother 63:767-70. https://doi.org/10.1093/jac/dkp026

115. Foulquier JB, Berneau P, Frérou A, et al (2019) Liposomal amphotericin B pharmacokinetics in a patient treated with extracorporeal membrane oxygenation. Med Mal Infect 49:69-71. https://doi.org/10.1016/j.medmal.2018.10.011

116. Zhao Y, Seelhammer TG, Barreto EF, Wilson JW (2020) Altered Pharmacokinetics and Dosing of Liposomal Amphotericin B and Isavuconazole during Extracorporeal Membrane Oxygenation. Pharmacotherapy 40:89-95. https://doi.org/10.1002/phar.2348

117. Watt KM, Gonzalez D, Benjamin DK, et al (2015) Fluconazole population pharmacokinetics and dosing for prevention and treatment of invasive candidiasis in children supported with extracorporeal membrane oxygenation. Antimicrob Agents Chemother 59:3935-3943. https://doi.org/10.1128/AAC.00102-15 